\title{
Strange Attractors: The Appeal of Chaos
}

$\mathrm{I}$

$\mathrm{T}$ is difficult to draw the line between what is genuinely new in the strange-attractor branch of chaos theory and what is the result of changed perceptions. Certain aspects of chaos theory were not previously known-that a meaningful distinction could be made between chaotic and disordered systems, for example, or that systems that appeared disordered could nevertheless manifest deep structures of order. From a scientific point of view, these discoveries perhaps justify calling chaos theory a new science (although, as we have seen, Poincaré's seminal papers date back to the I 890 s). From a broader perspective, however, the thrust of chaos theory comes as much from the realization that nonlinearity is pervasive throughout the natural world as from new theories as such. Where the eighteenth century saw a clockwork mechanism and the nineteenth century an organic entity, the late twentieth century is likely to see a turbulent flow. The importance of chaos theory does not derive, then, solely from the new theories and techniques it offers. Rather, part of its importance comes from its re-visioning of the world as dynamic and nonlinear, yet predictable in its very unpredictability.

At issue in any re-visioning is a shift in what is taken as the norm. If chaotic systems exist now, they obviously existed before. Turbulence, for example, has been an object of investigation in fluid mechanics for virtually as long as the field existed. But only in the last 


\section{The Figure in the Carpet}

few years has nonlinearity been recognized as representative rather than exceptional. Presumably observations confirming the extent of nonlinearity in the natural world could have been made at any time; the point is they were not. Similarly, scale dependence could have been observed in many phenomena before the emergence of the new paradigms. Although some examples were recognized, they were considered to be exceptions to the rule rather than to instantiate a rule. Also overlooked was the inability to specify initial conditions accurately enough to predict future states of the system. Although a wide variety of behaviors might have made this fact clear, the emphasis in Newtonian paradigms on long-term prediction directed attention away from the issue, making it seem like a nonissue. Joseph Ford, for example, points out that specifying initial conditions within physics has traditionally been regarded as "a task of such apparent triviality that no mention of it appears in the [technical] literature" (1983:44). The more subtle aspects of chaos theory have to do with changes in orientation and focus. For this reason, it may appear that the new science is simply discovering what everyone has known all along. There is some truth in this observation-but only some, for knowledge does not exist in an ideal space removed from culture. What is known is a function of what is noticed and considered important. If the criteria defining center and margin change, in a very real sense the structure of knowledge changes as well.

The power of a new paradigm to reinterpret well-known phenomena can be aptly illustrated through the swinging pendulum that is a textbook example of Newtonian mechanics. Suppose we construct a pendulum that consists of a ball at the end of a motorized shaft. Ordinarily the ball swings parallel to the driving momentum of the motor. However, if the frequency of the motor shaft's oscillations is close to the pendulum's natural period, the ball also develops a perpendicular motion. The interaction of this motion with the driving force causes the pendulum to become extremely sensitive to small variations in the starting conditions. In fact, a pendulum oscillating in this fashion has such sensitive dependence on initial conditions that it is impossible to measure the starting point accurately enough to ensure that the pendulum will swing the same way twice. David Tritton, who uses this device to demonstrate chaos in action, comments that if "we knew exactly how the pendulum is moving at a 
given time, then we could predict its future motion exactly. But we never do know anything exactly - the slightest vibration in the drive or the slightest draught in the room prevents that" (r 986:37). 'Thus the system that was emblematic of Newtonian determinism for the eighteenth century, a swinging pendulum, can easily be made to act unpredictably.

If chaos is everywhere, even in the deceptive simplicity of a pendulum, why was it not noticed before? Scientific journals are full of articles attributing departures from expected orderliness to errors, faulty experiments, or erratic equipment. Researchers interested in chaos theory are returning to these "noisy" data and testing them for the characteristic patterns they have learned to recognize; in a significant number of cases, the patterns are there. It seems clear that they were not noticed before because no paradigm existed through which they could be understood. In Chaos (I987), James Gleick documents how researchers in various fields slowly became aware that others were working along similar lines and that what they were seeing were not aberrations but a new kind of normality. The emergence of chaos is virtually a casebook demonstration that scientific investigation is not simply a matter of objectively describing nature.

What is it a matter of? Chaos theory is still so new that little work has been done on how it became a new paradigm. To my knowledge there are only two explorations of how this paradigm shift came about. One is an appendix to Benoit Mandelbrot's Fractal Geometry of Nature ( 1983 ), in which he argues that the mathematics associated with chaos was the work of mavericks who refused to follow received ideas. The second is Gleick's Chaos. Gleick, a science writer for The New York Times, concurs with Mandelbrot's view that chaos emerged from the work of a few solitary individuals. "No committee of scientists pushed history into a new channel," Gleick writes, "- $-\mathrm{a}$ handful of individuals did it, with individual perceptions and individual goals" (p. r82). I believe we should view such explanations skeptically, for they fit too neatly into the American

\footnotetext{
'Tritton's discussion is intended for a general audience. A more technical treatment is D. d'Humières, M. R. Beasley, B. A. Huberman, and A. Libchaber, "Chaotic States and Routes to Chaos in the Forced Pendulum," Physical Review A 26 (1 982 ): $3483-3496$.
} 
myth of the rugged individual who single-handedly opens up a new frontier. I shall return to Gleick's book to discuss how it inscribes scientific investigation into narrative patterns that imply science is an individual enterprise rather than a reenactment of highly charged cultural concerns. At issue is not only what chaos implies as a new paradigm but what it can tell us about the culture in which it is embedded. First, however, it is necessary to understand more about chaos in its scientific sense.

\section{The Deep Structure of Chaos}

Before chaos became a recognized research field, Edward Lorenz happened upon its fundamental properties when he was trying to model the earth's weather. Lorenz thought that he could capture the essence of how weather changes through three nonlinear differential equations. ${ }^{2}$ The nonlinearity was important, for it kept the patterns from repeating themselves exactly. However, Lorenz got more unpredictability than he bargained for. He had programmed his computer to display the solutions graphically, as wavy lines of symbols. Gleick tells the story of how Lorenz stopped a sequence in midcourse, and rather than start over from the beginning, typed the mid-point values into the computer to start the sequence running again (Gleick, 1987:15-18). Lorenz presumed that this mid-point run would match the first run exactly. To his surprise, when he compared the two lines he found that they diverged-at first only a little, but then more and more.

On reflection he realized that the display was amplifying a minute difference in initial conditions. The computer stored six digits in its

${ }^{2} \mathrm{~A}$ differential equation expresses the rate of change of one variable with respect to another; for example, how the pressure of a gas changes as the temperature is raised. If both variables change at the same rate (that is, if for every additional degree of temperature the pressure rises one unit), the graph expressing this relation will be a line tilted at a 45 -degree angle between the two right-angle axes. If the relation between the variables is nonlinear, the graph will show a curve rather than a straight line. If it is strongly nonlinear, the curve will have a very sharp hump (or humps) in it. Only with linear differential equations can one be sure of arriving at a general solution. Some nonlinear differential equations can be solved, but these are special cases. 
memory, but the printout displayed only three. When he started the mid-point run with these three digits, he was beginning from a very slightly different place than where the run had stopped. This small initial discrepancy was amplified until the final result was a pattern quite different from the original. Carried into weather forecasting, this result implied that a sea gull flapping its wings on a California beach could affect the occurrence of snow on Wall Street.

Fascinated, Lorenz began to explore the behavior of his model in more detail. He found that the data contained what would later be called a strange attractor. An attractor is simply any point within an orbit that seems to attract the system to it. A swinging pendulum, for example, eventually comes to rest at the mid-point of its period unless it is driven by a motor. Because this point is always the same, the pendulum is said to have a fixed-point attractor. Other oscillators have attractors that are cycles, such as the double rhythm of the human heart. When the heart is disturbed (provided the disruption is not too massive), it returns to this characteristic rhythm even if it begins from a different place. Because the cycle takes place within well-defined boundaries that limit the extent of variations within any given cycle, it is called a limit cycle. ${ }^{3}$

One way to model an attractor is to construct a phase space. Suppose we want to describe a swinging pendulum. For convenience we can choose one point in each swing and note what the pendulum is doing at that point. Say we choose the mid-point. Each time the pendulum passes the mid-point, we note its position and velocity. To see how these numbers change over time, we plot them on a graph, with the horizontal axis representing position and the vertical axis velocity (see figure 3 ). ${ }^{4}$ Rather than show the pendulum's actual motion as a movie would, this graph corresponds to a series of snapshots depicting the pendulum's behavior at a single instant in each cycle. If the pendulum is driven by a motor at a constant rate, the

${ }^{3}$ For a discussion of heart rhythm as a limit cycle, see Ary L. Goldberger, Valmik Bhargava, and Bruce J. West, "Nonlinear Dynamics of the Heartbeat," Physica $17 \mathrm{D}$ (1985): 207-244. Arthur T. Winfree also has an excellent discussion in When Time Breaks Down: The Three-Dimensional Dynamics of Electrochemical Waves and Cardiac Arrhythmias (Princeton: Princeton University Press, 1987).

${ }^{4}$ Douglas Hofstadter has a clear and accessible explanation of phase space in "Metamagical Themas," Scientific American 244 (November 1981): 22-43. 

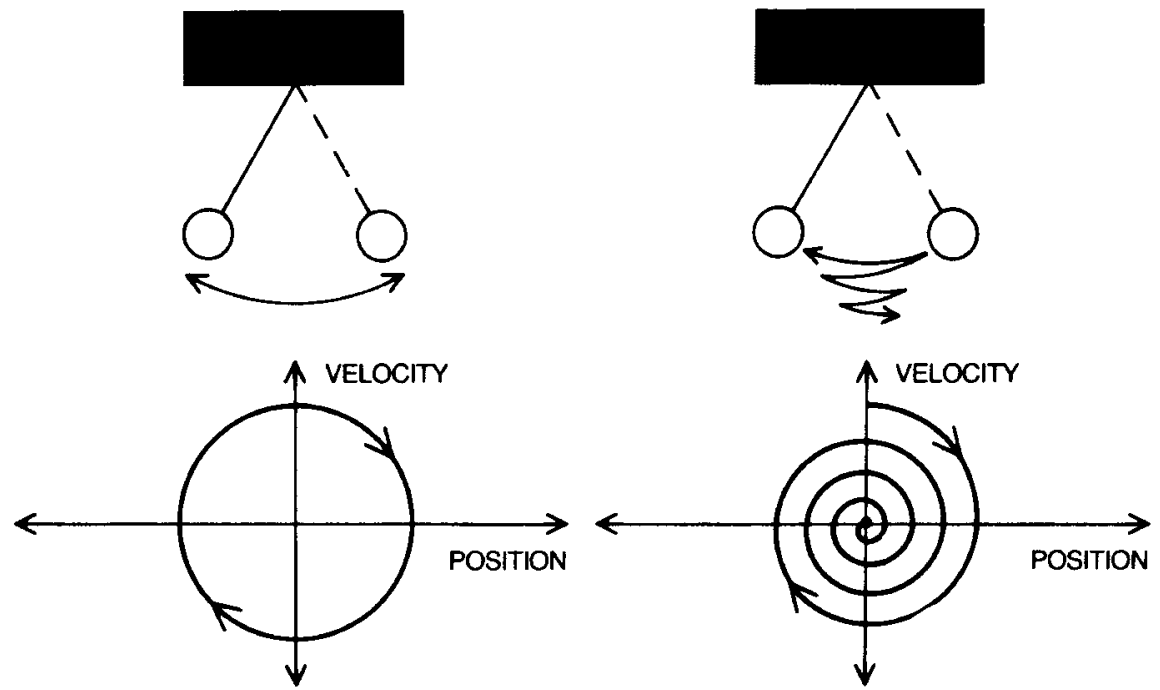

3. Phase space diagram of a pendulum being driven at a constant rate (left) and running down (right). Drawing by Andrew Christie from James P. Crutchfield, J. Doyne Farmer, Norman H. Packard, and Robert S. Shaw, "Chaos." Copyright (C) I 986 by Scientific American, Inc. All rights reserved.

graph will show a circle. If it is slowed down by friction, the diagram will show a spiral contracting toward the middle. Because there are two quantities that can vary-position and velocity-the system is said to possess two degrees of freedom, and the graph representing these variables is called a two-dimensional phase space.

It is important to remember that a phase space diagram is not the same as an ordinary drawing whose dimensions correspond to length, width, and depth. Phase space dimensions represent variables within the system, and phase space shapes show how they change over time. An orbit in phase space should thus not be confused with a trajectory through space. Rather, it is a map of the changes in the system's behavior over repeated cycles. Systems more complicated than pendulums will have more degrees of freedom and consequently will require greater dimensions for their representation. When the degrees of freedom exceed three, these systems cannot be easily drawn. Their mathematical properties, however, can still be explored.

Recall that Lorenz's model was represented by three nonlinear differential equations, corresponding to three degrees of freedom. 


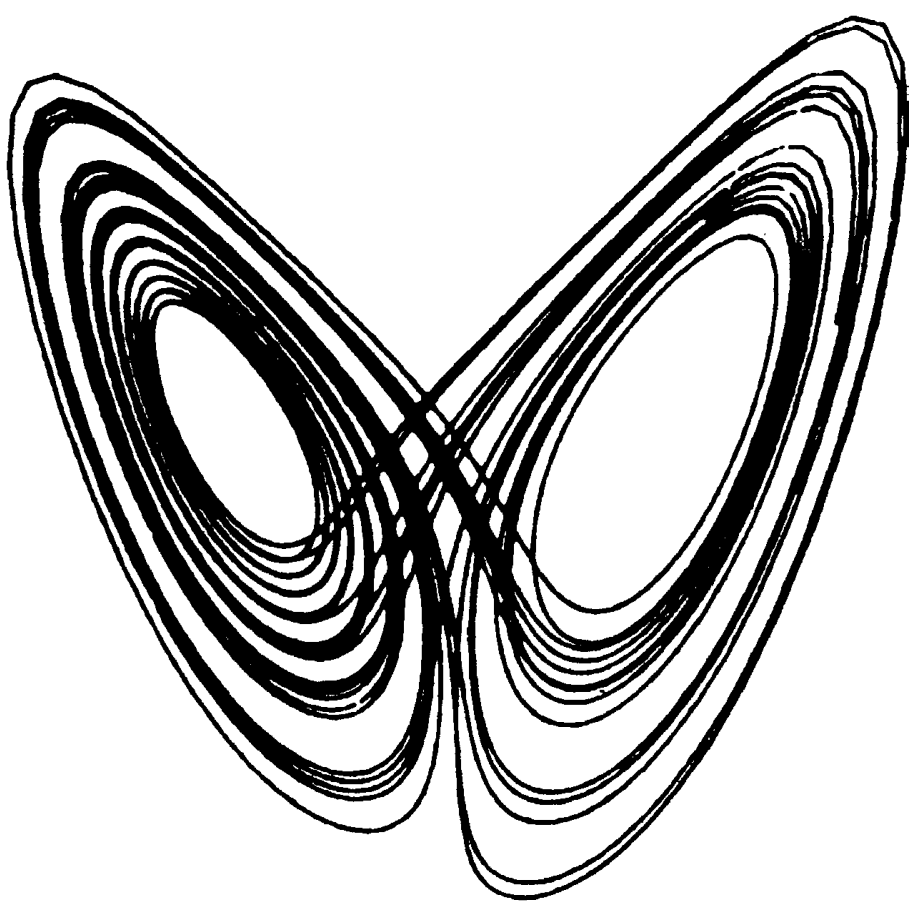

4. Phase space diagram of a Lorenz attractor. Courtesy of Herbert Hethcote.

When he graphed its motion in a three-dimensional phase space, he saw a shape resembling two butterfly wings, intricately traced by the orbits as they repeated the cyclic motion over and over (figure 4 )..$^{5}$ The orbits always stayed within a certain volume, so the shape was quite distinctive; but within this volume, no two orbits ever intersected or coincided, an indication that the system never repeated the same motion exactly. Because the orbits "wandered" in complex and unpredictable ways, knowing the starting point for a given run did not help one to know where the system would be at any future moment. Starting coordinates that were very close on the number scale could lead to orbits far apart; conversely, points numerically far apart might end in orbits close together. No wonder this attractor was considered strange. It was an odd combination of simplicity and complexity, determinism and unpredictability.

${ }^{5}$ See Lorenz, 1963, and his "Problem of Deducing the Climate from the Governing Equations," Tellus I6 (I964): I-II. 
What gives a phase space these peculiar properties? Drawing on early work by the distinguished mathematician Steven Smale, theorists realized that oscillators that behaved in this fashion acted as though the phase space within which they moved had been squeezed and then folded over, much as croissant dough is rolled out and folded over itself again and again. ${ }^{6}$ This process creates an extremely complex interleaving of very thin layers. If it is carried on long enough, the layers approach infinite thinness, just as orbits in phase space have zero thickness. Nevertheless, each orbit remains distinct from every other; no two ever exactly coincide. James Crutchfield and his collaborators (1986) simulated this process through the computer manipulation of an image (they impishly chose a portrait of Henri Poincaré, the founder of dynamical systems theory). The simulation showed the outlines of Poincaré's face quickly becoming unrecognizable as the points spread out. But after a certain number of squeezings and foldings, the points aligned themselves so that the image briefly reappeared, as though the memory of the intact image lingered within the system (see figure 5). This odd combination of randomness and order conveys the flavor of a strange attractor.

The force of chaos theory derives from the discovery that an astonishing variety of systems can be modeled as strange attractors. For example, they have been shown to describe outbreaks of infectious diseases, variations in cotton prices and in the numbers of lynxes caught by trappers, the rise and fall of the Nile River, and erratic eye movements in schizophrenics.' Gleick reports that the group of graduate students at Santa Cruz who became pioneers in the chaos theory played a coffeehouse game of guessing where the nearest strange attractor was: in the din of dishes coming from the kitchen? in the swirl of cream in coffee? in the clouds of cigarette smoke coming from the next table (I987:20I-226)? According to

\footnotetext{
${ }^{6} \mathrm{~A}$ discussion of this process is presented in Crutchfield et al., 1986. Steve Smale gives an account of his work in The Mathematics of Time: Essays on Dynamical Systems, Economic Processes, and Related Topics (New York: Springer, 1980).

${ }^{7}$ See, for example, William M. Schaffer and Mark Kot, "Nearly One-Dimensional Dynamics in an Epidemic," Journal of Theoretical Biology I I 2 (I985): 403-427; William M. Schaffer, "Stretching and Folding in Lynx Fur Returns: Evidence for a Strange Attractor in Nature?" American Naturalist I 24 (1984): 798-820; Bernardo A. Huberman, "A Model for Dysfunctions in Smooth Pursuit Eye Movement," Xerox preprint, Palo Alto Research Center.
} 

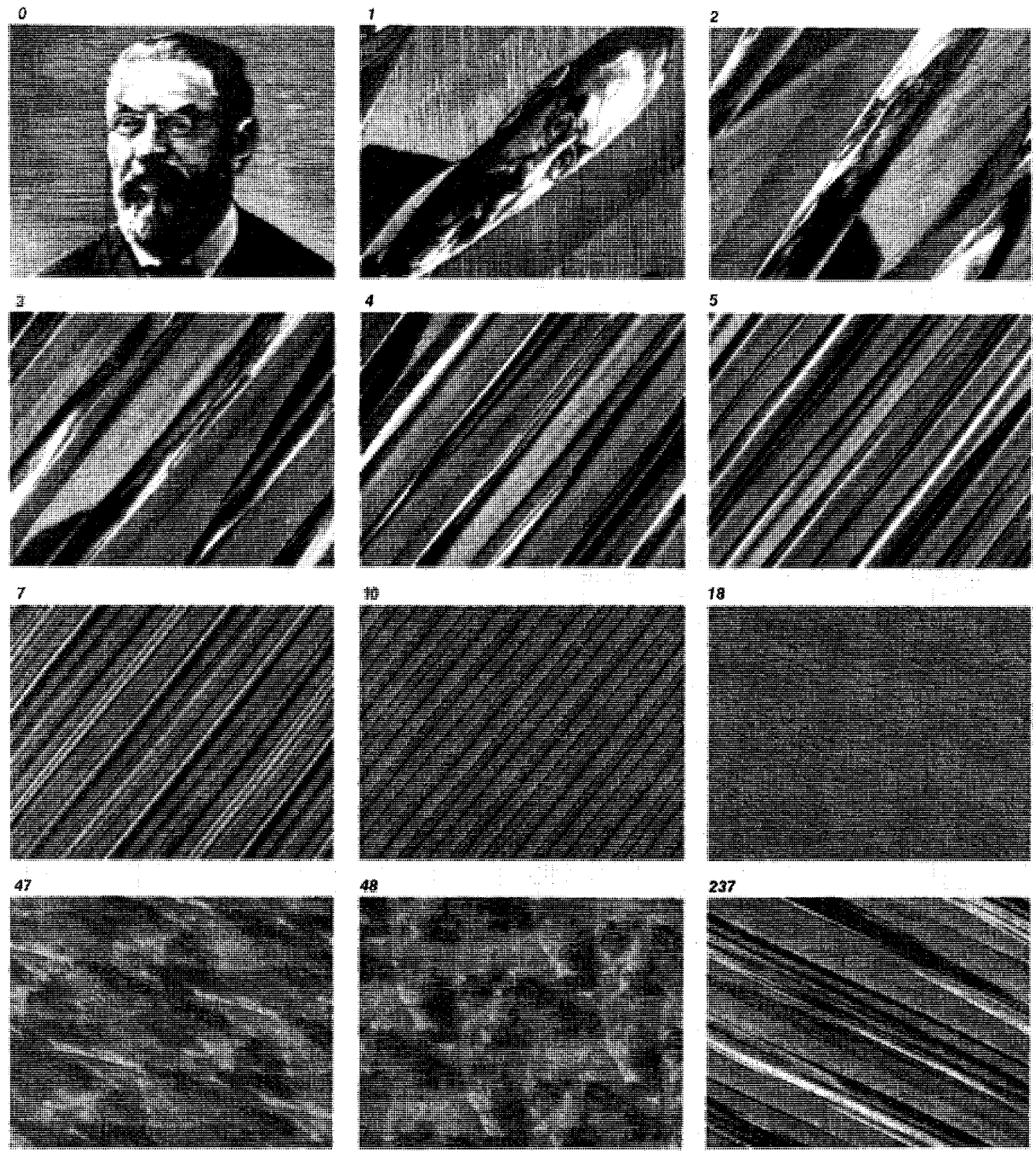

239

240
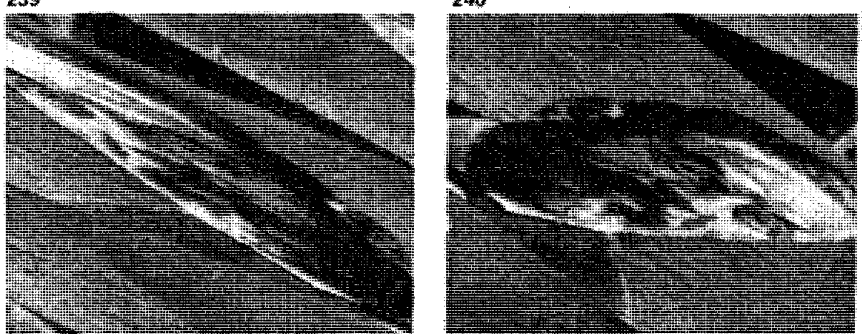

241

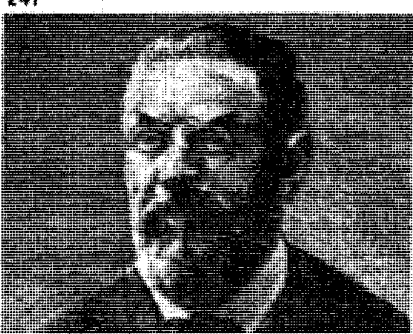

5. Stretching an image of Henri Poincaré. The number above each box indicates the number of times the stretching operation has been performed. Reproduced from James P. Crutchfield, J. Doyne Farmer, Norman H. Packard, and Robert S. Shaw, "Chaos," by permission of James P. Crutchfield. Copyright $(\mathbb{C}$ I 986 by Scientific American, Inc. All rights reserved. 
Gleick, a faculty member at Santa Cruz became convinced that his rattling speedometer was a strange attractor. Everywhere they have looked, chaologists have found seemingly erratic variations that prove to have the deep structure of chaos.

What does it mean when systems with so little in common as fur trapping and measles epidemics, eye movements and cotton prices, follow the same model? What has one understood when one creates a model that pays no attention to the specific mechanisms at work in the system? Do such models count as explanations? Do they even count as science? To begin exploring these questions, I turn to the work of Mitchell Feigenbaum, who perhaps more than anyone else deserves credit for recognizing the universal nature of chaos.

\section{Chaos as Universality Theory}

One approach to chaos is to consider the closely related concept of randomness. In a review article in Los Alamos Science (I980), Feigenbaum asks us to consider in what way a number generated by a computer can be random. He points out that a computer-based random number generator can easily be constructed by means of a program that does "nothing more than shift the decimal point in a rational number whose repeating block is suitably long" (p. 4). Numbers generated by this method are so lacking in pattern that they satisfy the most rigorous tests for randomness, yet the method that produces them is perfectly simple and deterministic. Technically, these numbers are called pseudo-random to indicate they have been generated by a deterministic computer program. Feigenbaum's inspiration was to wonder whether other apparently chaotic phenomena also might be pseudo-random, obeying deterministic programs just as pseudo-random numbers do. This astonishing premise amounts to saying that chaos has a deep structure.

One of the first indications a deep structure might in fact exist was Feigenbaum's discovery that systems that go from ordered to chaotic states follow a characteristic pattern of period doubling. Let us say that we are looking for a pattern in the behavior of an electrical oscillator. We notice the oscillator repeats its behavior after some time interval $T$. As the temperature is raised, the oscillator's behav- 
ior becomes more erratic, and we now have to extend the time period to $2 T$ to have a repeating pattern. When the temperature is raised yet again, the time required to observe a repeating pattern jumps to $4 T$, and so on. Eventually the time period will become so great that the oscillator has no repetitions in the time scale available for observation. At this point it is said to be chaotic. Period doubling is now recognized as a powerful generalization, describing the onset of chaos in everything from dripping faucets to Niagara Falls. ${ }^{8}$ At the time of his discovery, however, Feigenbaum was not thinking about physical systems. He was looking at the behavior of mathematical functions when they are iterated.

To iterate a function means to use the output of one calculation as input for the next, each time performing the operation called for by the function. It is analogous to beginning at a certain place and doing a dance step; then starting from the new location each time, doing the dance step again and again. Iterating strongly nonlinear functions produces paths that have folds in them when they are displayed in phase space. The folded orbits Feigenbaum produced were simple one-dimensional maps. The behavior of these iterated functions, however, was analogous to the more complex stretching and folding of multidimensional phase space which is characteristic of strange attractors. "This general mechanism," Feigenbaum comments, "gives a system highly sensitive upon its initial conditions and a truly statistical character: since very small differences in initial conditions are magnified quickly, unless the initial conditions are known to infinite precision, all known knowledge is eroded rapidly to future ignorance" (p. 2I).

The startling aspect of Feigenbaum's work was his discovery that despite the different operations performed by different nonlinear functions-despite the different dance steps they used-their iterated paths approached chaos at the same rate and showed the same characteristic patterns of period doubling. All that mattered was that the paths had folds of sufficient steepness. It may be hard for a nonscientist to appreciate the enormity of this discovery, but to a mathematician, sine waves are as different from quadratic equations as

\footnotetext{
${ }^{8}$ Robert Shaw, The Dripping Faucet as a Model Chaotic System (Santa Cruz: Aerial, 1984). Niagara Falls would of course be an instance of turbulent flow, as discussed in Wilson, 1983 .
} 


\section{I54 The Figure in the Carpet}

pirouettes are from bows to a ballet dancer. To find out that there is a way of looking at these functions that makes their operations seem not just similar but identical is analogous to discovering that there is a way of looking at Nureyev dancing and Donald Duck waddling that makes their performances into a universal constant applicable to anyone moving on that stage.

What was this new way of looking? At the same time that the iterative process had the effect of overwhelming individual differences between functions, it also revealed a universality in the way large-scale features related to small details. The shift in focus was from the particularities of a given function to the relation between different recursive levels in the iterative process. Imagine two paintings, each showing an open door through which is revealed another open door, through which is another and another. ... One way to think about the doors in these two paintings is to focus on the particularities of the repeated forms. Suppose the doors of the first painting are ornately carved rectangles, whereas the second painting shows doors that are unadorned arches. If we attend only to shapes, the paintings may seem very different. But suppose we focus instead on the recursive repetition and discover that in both paintings, the doors become smaller at a constant rate. Through this shift in focus we have found a way of looking at the paintings that reveals their similarity to each other and to any other painting constructed in this way. The key is recursive symmetry.

\section{Chaos and Symmetry}

The mathematics Feigenbaum used to reveal recursive symmetries was developed by Kenneth Wilson, winner of the 1982 Nobel Prize in physics. To understand Wilson's approach, consider what happens when a flow becomes turbulent (Wilson, r983). Often microscopic fluctuations within a flowing liquid cancel each other out, as when a river flows smoothly between its banks. In this case each water molecule follows much the same path as the one before it, so that molecules starting close together continue to be close. Sometimes, however, microscopic fluctuations persist and are magnified up to the macroscopic level, causing eddies and backwaters to form. 
Then molecules that began close together may quickly separate, and molecules that were far apart may come close together. As a result it becomes extremely difficult to calculate how the flow will evolve. "Theorists have difficulties with these problems," Wilson explains, "because they involve very many coupled degrees of freedom. It takes many variables to characterize a turbulent flow or the state of a fluid near the critical point" (p. 583). Indeed, the mathematics of turbulence is so complex that even the new supercomputers are inadequate to handle it. Computation times become unreasonably long after only three or four variables are considered, whereas dozens are necessary to create a model that can simulate turbulence accurately. Hence the importance of being able to solve these problems analytically.

Wilson's contribution was to devise a method that would sometimes yield analytical solutions. The essence of his approach was to shift from following individual molecules to looking for symmetries between different scales. In turbulent flow, for example, large swirls of water have smaller swirls within which are still smaller swirls. .. . To model these recursive symmetries, Wilson used renormalization groups. Renormalization had first emerged as a technique in quantum mechanics; physicists used it to get rid of infinite quantities when they appeared in the equations. Originally the only justification for the procedure was that it made the answers come out right. If you think this sounds suspiciously aribitrary, you are not alone. For years virtually all mathematicians and some physicists regarded renormalization as no more than hand-waving. But Wilson saw its deeper implications.

He knew that in renormalization certain quantities regarded as fixed, such as particle mass, are treated as if they were variable. He realized that there is a sense in which this is a profound truth rather than an arbitrary procedure. For example, we tend to think of a golf ball as a smooth sphere. But to a mosquito it would appear as a pocked irregular surface, and to a bacterium as the Wilson Alps. Renormalization implied that the choice of ruler used to measure physical properties affected the answer. At the same time, it revealed that there was something else-something not normally considered-that remained constant over many measurement scales. This was the scaling factor. By combining the renormalization process 
with the idea of a mathematical group, Wilson arrived at a method whereby this factor could be defined and calculated.

"Group," as it is used in mathematics, denotes a set of objects that is invariant under symmetry operations. For example, a cube rotated 90 degrees in any direction appears unchanged in its spatial orientation; it is therefore said to be invariant under right-angle rotation. Tetrahedrons are in the same mathematical group as cubes because they also have this property. The purpose of finding a renormalization group is to look for symmetries that are invariant for different measurement scales. As an illustration of one of these symmetries, consider the classic "middle third" set, first proposed by Georg Cantor in the nineteenth century. Imagine that we draw a line from $\circ$ to $\mathrm{I}$, as in figure $6 .{ }^{9}$ Now we erase the middle third of line $a$. Each of the smaller lines of $b$ has the same form as line $a$, and multiplying one of these line segments by 3 gives the original line back again. If we erase the middle third of the small lines of $b$, we create the even smaller lines in $c$. Each of the two broken lines of $c$ has the same form as the entire interval of $b$, and multiplying either segment by 3 gives line $b$ back again. If we keep erasing middle thirds, each time the symmetry of the resulting small part mirrors the larger part of the step before, and each time we can obtain the larger part by multiplying the smaller part by 3 . Sets that have this kind of symmetry are said to possess fixed points. The purpose of defining a renormalization group is to discover the operations and variables that allow fixed-point symmetry to emerge.

Groups that display fixed points have physical significance because the symmetry allows coupling to take place between different levels. When a system possesses fixed points, perturbations on the smallest scale are quickly transmitted throughout the system, affecting even the largest macroscopic level. Imagine a bullwhip moving at just the right frequency so that a small twitch of the handle is transmitted into larger and larger waves all the way to the end, causing the whip to emit a loud, satisfying CRACK. This kind of transmission and magnification is possible because a system possesses an appropriate kind of symmetry. When fixed-point symmetry is present, systems "crack" because the symmetry permits microscopic changes to translate into coordinated movements all through the system.

\footnotetext{
9Cantor's "middle third" set is discussed at length in Mandelbrot, $1983: 74-96$.
} 
a.

0

1

$b$.

0

1

c.

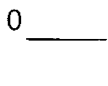

6. Cantor's "middle third" set

We can appreciate the generality of this approach by looking at the range of behavior to which it applies. As we have seen, one area is turbulent flow; another is phase change behavior, as when water turns to steam. The transition from water to steam illustrates how fluctuations at the microscopic level can be translated into dramatic macroscopic changes. Water and steam appear to be very differentone is liquid, the other gas. However, this gross change in macroscopic properties originates in microscopic changes. At the point where the phase change takes place, the system is characterized by "bubbles of steam and drops of water intermixed at all size scales from macroscopic, visible sizes down to atomic scales" (Wilson, I $983: 583)$. At this critical point, and only at this point, the system undergoes a phase change because it possesses the fixed-point symmetry that allows changes at the smallest level to be transmitted all the way up to the largest level. Thus turbulent flow and phase change behavior, different as they are, can both be understood in terms of the scaling symmetries. Additional applications are in quantum field theory, where coupling mechanisms between particles are important.

To see how this approach can be applied to chaos, consider it as an explanation for strange attractors. A strange attractor possesses fixed-point symmetry, implying it has very many coupling points that transmit and magnify tiny fluctuations into large changes within its orbit. The physicist M. V. Berry has likened this situation to playing tennis in an orchard." Two successive balls may be hit in very nearly identical ways, yet, they will wind up in widely separated locations within the orchard because each time they are deflected by

\footnotetext{
${ }^{10}$ M. V. Berry, "Regular and Irregular Motion," in Jorna, I 978:16-I 47.
} 
a tree, their trajectory changes. The more trees there are for the balls to hit, the more impossible it is to predict where they will end up. In this analogy, the trees correspond to coupling points, and the final location of the balls to points along a strange attractor's orbit after several iterations. We may know fairly precisely in what direction a ball is first hit; that is, we know its initial conditions. But unless we know exactly what the direction is-unless, as Feigenbaum pointed out, we know the initial conditions with infinite precision-we will not be able to hit a second ball so that it falls even close to the first. After a relatively short time, balls will be scattered throughout the orchard at locations that cannot be calculated in advance. Thus deterministic systems may also be chaotic.

One limitation of Wilson's method was its inability to tell whether a system possessed fixed points. If fixed points were there, it could yield an analytical solution; but it could not predict whether a solution was possible. Nevertheless, it was a breakthrough because it synthesized previous research into a single explanatory framework. And it gave Feigenbaum an approach to use in his efforts to understand chaos.

Whether Feigenbaum's work was a startling discovery or an extension of what was already known continues to be debated. Some scientists see Feigenbaum's universality theory as central to the science of chaos. Others regard it as derivative, especially since Feigenbaum's scenario of period doubling emerges as a special case of Wilson's group symmetries. Critics say that Feigenbaum was looking at only a narrow spectrum of chaotic behavior, and that work by Wilson and others was more powerful and general. Feigenbaum's concentration on iterative equations rather than physical models is also an issue; it was only when the same behavior was found in experimental data that chaos became a major research area. Nevertheless, Feigenbaum was the first to realize that scaling symmetries implied not just qualitative similarities but exact numerical correspondences. He was the first to see that chaos, in its way, had a structure as rigorous and compelling as order.

\section{Chaos as Maximum Information}

One of the curious chapters in the history of chaos is the role played by a group of graduate students at the University of Califor- 
nia at Santa Cruz. Gleick reports how the group formed around Robert Shaw, constituted themselves as a collective, and began a research program that mingled investigations into chaotic systems with a fanatic interest in roulette (I987:20I-226). As members of the collective tell it, they worked virtually alone; their advisers in the physics department neither approved nor understood the significance of what they were doing. Although each member made important contributions, Shaw is perhaps the best known. It was his idea to connect chaos with information.

In his article "Strange Attractors, Chaotic Behavior, and Information Flow" (I98I), Shaw analyzes strange attractors by dividing their orbits into minimum blocks of phase space, with dimensions on the order of Planck's constant (the very small number that appears as a universal constant in the uncertainty principle). This minimum space is identified with a "logon" or information cell of a minimum size, thus translating the oscillator's orbit into a flow of information. Having the flow contract, as it does when an oscillator begins outside its limit cycle, is equivalent to saying that information once accessible has been destroyed. With strange attractors the reverse process takes place; information that was once inaccessible is created. Attractors operate irreversibly because their operation changes what we can know about them, not merely what we do know.

Shaw's analysis of an attractor's orbit as a flow of information creates a perspective fundamentally different from that of classical Newtonian dynamics. Unlike Newtonian paradigms, it does not assume that quantities are conserved. Information is not merely transformed, as matter and energy are. It really does come into being or disappear. Once an oscillator with a contracting information flow has made the transition to its steady state, the information contained in the initial conditions cannot be recovered. Conversely, knowing only the initial conditions and the relevant equations of motion will not allow one to predict where an oscillator with an expanding flow will be at some future time.

Since the equations governing a strange attractor's motion are deterministic, where does its unpredictability come from? Recall that Feigenbaum located the source of uncertainty in the initial conditions. He argued that this was the only place uncertainty could have entered, because the equations and the computer iteration were both 
deterministic. Shaw's focus on physical systems, by contrast, leads him to an experimentally based interpretation. He sees the chaos as coming from "hitherto unobservable information, such as a random fluctuation of the heat bath, [which] has been brought up to macroscopic expresssion" (p. 84). In this view the oscillator acts as a "translator" between the microscopic and macroscopic levels. Because the uncertainty principle guarantees that fluctuations always exist on the particle level, there will always be enough microscopic uncertainty to initiate macroscopic chaos. All that is required is for the system to have the appropriate symmetry so that it can act as a translator. Thus Shaw asserts that Newtonian determinism is "not only wrong in the small, where quantum mechanical uncertainty limits our knowledge of the exact state of the system, but it is wrong in the large" (p. 108).

The celebratory tone of his conclusion is strikingly different from the cautious sobriety found in most scientific journals. For Shaw chaos is the source of all new information in the world. He revels in the implication that chaotic systems are much more abundant in nature than ordered ones. Envisioning the physical world as a great swirling flow of information, he sees new information "continuously being injected ... by every puff of wind and swirl of water." He acknowledges that this "constant injection of new information into the macroscales may place severe limits on our predictive ability." More important for him, however, it "insures the constant variety and richness of our experience" (p. Io8). Shannon would have approved. Thirty years earlier he had intuited that chaos was information's partner. Even he, however, did not imagine that their coupling could bring forth the infinite fecundity of nature.

\section{Chaos and the Limitations of the Human Mind}

Despite Shaw's eloquent conclusion, not everyone sees infinite information as cause for celebration. Joseph Ford, a flamboyant physicist at Georgia Institute of Technology who runs an informal clearinghouse for chaos papers, argues that infinite information is essentially inhuman ( 1983 ). He believes that numbers harboring infinite complexity should be excluded from numerical representation 
because they exceed the ability of humans to understand them. His concern centers on results from algorithm complexity theory demonstrating that almost all real numbers are infinitely complex. When even such seemingly well-behaved creatures as real numbers are capable of chaotic behavior, they bewilder the humans who conceived them.

To understand Ford's concern, it is necessary to know something of algorithmic complexity theory. An algorithm is the set of procedures a computer uses to solve a problem, such as the instructions telling a computer to generate random numbers by moving the decimal point in a rational number. Algorithmic complexity theory is concerned with defining and calculating the amount of information an algorithm contains. The theory, invented independently by Gregory Chaitin (then an undergraduate at City University of New York) and A. N. Kolmogorov of the Academy of Sciences of the USSR, was proposed to solve the problem of how a number can be named (Chaitin, 1975).

The problem of how to name a number may seem trivial until one considers an example known as Berry's Paradox, first published in Principia Mathematica and reviewed by Martin Gardner (I979). Consider the number "one million, one hundred one thousand, one hundred twenty-one." This number appears to be named by the expression "the first number not namable in under ten words." However, this definition has only nine words. Is the number named by its nine-word definition, which says that the number cannot be named in under ten words? As Gardner points out, the paradox demonstrated that naming is too powerful a concept to be used without restriction (1979:20). To resolve it and similar paradoxes, algorithmic complexity theory restricts the concept of naming by saying that an integer has been named when it has been calculated by a computer program. Such a program then becomes the number's name. Algorithmic complexity theory defines the complexity of this name as the bit length (that is, the amount of information) of the shortest computer program that can print the given number sequence. Maximum complexity is achieved when the computer program generating the number is not appreciably shorter than the number itself.

To see how this idea relates to chaos, recall that, by definition, 
random numbers have no pattern to their sequences. Thus the algorithm generating a random number $n$ might read, "Print $n$." But specifying $n$ in this program takes about as much space as $n$ itself, because the information contained in the number cannot be appreciably compressed. Thus the simplest way to specify the sequence is to provide a copy of it. Chaitin shows that almost all real numbers are random in the sense that they cannot be generated by a finite computer algorithm (1975:50). Following Mark Kac, Ford points out that "the [number] continuum therefore has the distinction of being a well-defined collection of mostly undefinable objects" (1983:44).

What does this discovery mean for human knowledge? Ford connects it with the importance chaos theory places on specifying initial conditions. As we know, unless the initial conditions are stated with infinite precision, the smallest uncertainty in a strange attractor can quickly translate into macroscopic chaos. Because most real numbers are random, however, they cannot be stated with complete accuracy. Thus unpredictability in deterministic systems is inevitable. In Ford's view, this realization will force a reformulation "whose impact on science may be as significant as [relativity theory and quantum mechanics]" (1983:46).

Ford's suggestions for the direction this reformulation might take are based on his belief that humans cannot cope with infinite complexity. He proposes that we move toward a "humanly meaningful" number system by eliminating numbers with infinite information content. First to go are the "totally inhuman numbers" that possess maximum complexity, numbers that "require infinite information to compute, to store and to define." Next are the "seemingly innocent" numbers computed by repeating a finite algorithm an infinite number of times. Last to be excluded are the infinitely large $(1+1+1$ ...) and the infinitely small $1 /(1+1+1 \ldots)$. These successive eliminations would leave the number continuum so riddled with holes that Ford predicts all physical variables would henceforth be quantized (1983:47).

How far this view is from Shaw's celebration of chaos is apparent in the fable with which Ford ends his article. It is a mathematical version of the fall from Paradise. Man, at first content with simple integers, succumbs to temptation when he accepts an infinite num- 
ber from his mate. The man's mind "reached and fleetingly grasped the meaning of $(1+1+1 \ldots)$, but by morning he retained only the empty symbols" (1983:47). Ford thus implies that from a human viewpoint, infinite information is indistinguishable from total incomprehensibility, a conclusion Borges anticipated in "The Library of Babel." One is accustomed to encountering such thoughts in Ficciones. What does it mean to read them in Physics Today?

\section{The Beauty of Chaos}

Many scientists working on chaos speak of the need to "develop intuition." "They point to the fact that most textbooks treat linear systems as if they were the norm in nature. Students consequently emerge from their training intuitively expecting that nature will follow linear paradigms. When it does not, they tend to see nonlinearity as scientifically aberrant and aesthetically ugly. But nonlinearity is everywhere in nature and consequently in mathematical models. Despite its prevalence, it has been ignored for good reason: except in a few special cases, nonlinear differential equations do not have explicit solutions.

A key factor in getting around this difficulty has been the development of microcomputers, which has led to a new style of mathematics. Ordinarily one does mathematics by stating a theorem and developing a proof. Mathematical reasoning thus proceeds through theorem-proof, theorem-proof, theorem-proof. But with computers, a new style of mathematics is possible. The operator does not need to know in advance how a mathematical function will behave when it is iterated. Rather, she can set the initial values and watch its behavior as iteration proceeds and phase space projections are displayed on a computer screen. Then she can see how the display modulates as she changes the parameters. The resulting dynamic interaction of operator, computer display, and mathematical functions is remarkably effective in developing a new kind of intuition. It is

\footnotetext{
${ }^{11}$ See, for example, Robert M. May and George F. Oster, "Bifurcations and Dynamic Complexity in Simple Ecological Models," American Naturalist i Io (1976): 573-599; and Benoit Mandelbrot, "Fractals and the Rebirth of Iteration Theory," in Richter and Peitgen, I986:15 I-I60.
} 
perhaps the scientific equivalent of performance art. Whereas Ford saw the computer as purveying inhuman complexity, one could argue that computer displays of fractals and dynamic systems make complexity intuitively meaningful. ${ }^{12}$

The foremost advocate of a new aesthetic appreciation of nonlinear mathematics is Benoit Mandelbrot. In his quirky and fascinating book, The Fractal Geometry of Nature (1983), he argues that thinking that nonlinearities are strange is itself strange, since complex figures appear regularly in nature. He compiles adjectives that other mathematicians have used to describe nonlinear geometry"monstrous," "counter-intuitive," "pathological," "psychopathic" -in much the same spirit as a Jesuit catalogues arguments refuting the existence of God, as an encyclopedia of misperception and error. Mandelbrot insists that on the contrary, highly complex and irregular forms are entirely compatible with our intuition and as beautiful as Nature herself. This beauty has been misperceived as "monstrous" because traditional geometry is ill equipped to deal with its complexities. "Many patterns in Nature," Mandelbrot writes, "are so irregular and fragmented, that, compared with Euclid... Nature exhibits not only a higher degree but an altogether different level of complexity" (p. 2).

To deal with the complexities of these forms, Mandelbrot invented fractal geometry. At its heart is the idea of a fractional dimension. Whereas Euclidean shapes can be represented very well by the familiar integer dimensions of a Cartesian space, irregular forms cannot. The corrugations that mark their surfaces give them, in effect, an added fraction of a dimension. Mandelbrot points out that the idea of fractional dimensions can also be applied to strange attractors. Earlier I compared the fixed-point symmetry of a strange attractor with a cracking bullwhip. Now I can refine that analogy by noting an important difference between an attractor and a whip. For the whip to crack, the length of the wave traveling down it must be such that an even number of wavelengths can fit into the total whip length. A strange attractor, by contrast, characteristically has an or-

${ }^{12}$ This aspect of computer technology is discussed in David Campbell, Jim Crutchfield, Doyne Farmer, and Erica Jen, "Experimental Mathematics: The Role of Computation in Nonlinear Science," Communications for the Association of Computing Machinery 28 (1985): 374-384. 
bit in phase space which is a fractional dimension of that space. If an attractor moves in a two-dimensional phase space, for example, its orbit may have dimensions of two and two-thirds. Every known strange attractor has this property. Just as the corrugations on complex surfaces impart an added fraction of a dimension, so the extremely complex wanderings of strange attractors' orbits give them fractional dimensionality. The same principle applies to other complex trajectories. Planets that move in regular paths have orbits that are integers of phase space, for example, whereas irregularly moving bodies such as asteroids have orbits of fractional dimensions. ${ }^{13}$

Strange attractors and wandering asteroids are a sideline for Mandelbrot. His main interest is fractal geometry, which he more or less invented by exploring forgotten paths and labyrinthine turns in earlier mathematics. He coined the word "fractal" from the Latin adjective fractus (meaning "broken") and fractional; it connotes both fractional dimensions and extreme complexity of form. An important difference between fractal and Euclidean geometry is the scaledependent symmetries of fractal forms. In Euclidean geometry, and in classical physics generally, Nature is considered "conformable to itself," of uniform consistency, so that what is true on one level is also true on another. In Euclidean geometry, for example, one equilateral triangle is taken to be similar to any other equilateral triangle, regardless of their relative sizes. For a geometry built on this assumption, the convoluted orbits of strange attractors look very strange, if not altogether deviant. But in fractal geometry the emphasis on recursive symmetries makes such orbits appear natural, because they are created by iteration of the same form over and over.

The complex couplings between scales of different lengths which are at the center of fractal geometry are found everywhere in nature-in cloud forms, mountain contours, tree grains. For these and many other natural forms, Mandelbrot explains, "the number of scales of length . . . is for all practical purposes infinite." Hence the importance of a geometry designed to elucidate transitions between levels by investigating and describing the symmetry operations that

${ }^{13}$ A related discussion is in Jack Wisdom, "Meteorites May Follow a Chaotic Path to Earth," Nature 3 I 5 (I985): 73 I-733. 
make them possible. So complex are these forms that it is extremely difficult to duplicate them precisely, even by Mandelbrot's techniques. Thus one speaks of approaching the natural form, rather than representing it exactly. Because of this complexity, Mandelbrot stresses that "the most useful fractals [i.e., those found in nature] involve chance and both their regularities and their irregularities are statistical." Scaling, as Mandelbrot uses the term, does not imply that the form is the same for scales of different lengths, only that the degree of "irregularity and/or fragmentation is identical at all scales" (1983:2).

In his richly illustrated text, Mandelbrot encourages readers to see computer-generated fractal figures as natural contours, pointing out that one resembles a mountain range, another a seacoast, still another the branching streams of a watershed. The comparisons are not merely fanciful, for much of the usefulness of fractals lies in their ability to model such naturally complex forms as the human vascular system and the lumps and clusters of galactic star systems. One of the figures that Mandelbrot discusses is Cantor's "middle third" set, previously mentioned as an example of fixed-point symmetry. When the diagram given earlier in figure 6 is extended into increasingly fine divisions, the resulting figure, called "Cantor dust," can be used as a model for the distribution of galaxies in the universe. When the horizontal lines of figure 6 , extended for several more levels, are made to flow vertically into one another to form two "draped" curtains, the figure models the breaks in Saturn's rings (1983:74-83).

Mandelbrot's procedure for generating fractals can be illustrated through the "Koch curve," named after its inventor, Helge von Koch. Imagine an equilateral triangle; suppose that we place smaller triangles, one-third the size of the original, on each side. We now have a hexagonal star, a Star of David. Suppose that along the sides of each point we place additional triangles reduced by one-third. Then on each of those triangles we place further triangles, again reduced by one-third, for as far as we wish to go (see figure 7). The increasingly complex curve traced by the boundary of this figure is obviously symmetrical, yet also possesses a complexity that approximates the irregular symmetries of a coastline, as can be seen in figure 8. 

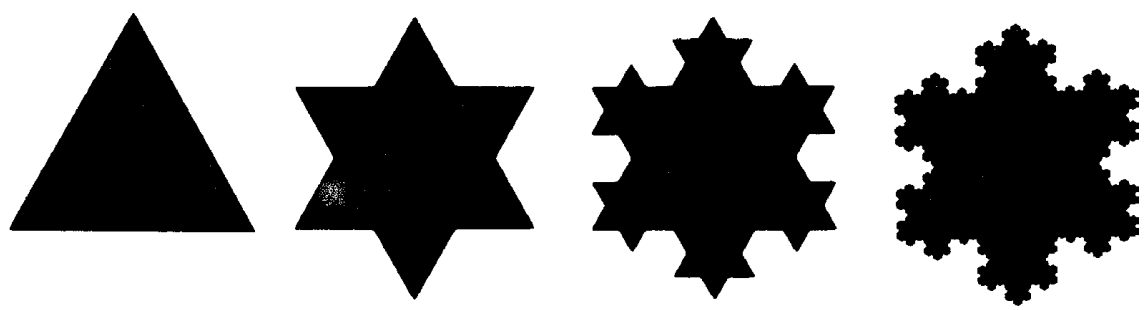

7. Generating a Koch curve. From Benoit B. Mandelbrot, The Fractal Geometry of Nature (New York, 1983), p. 47, by permission of W. H. Freeman Company.

The problem with such figures is that their complexities are too regular to fit most natural objects. We can improve the fit by "tampering" with the program to introduce a statistical element. Why introduce chance instead of generating more complexities deterministically? Because the objects to be modeled, such as coastlines, are themselves formed by chance. A deterministic approach "would be not only tedious, but doomed to failure," Mandelbrot argues, "because each coastline is molded through the ages by multiple influences that are not recorded and cannot be reconstituted in any detail. The goal of achieving a full description is hopeless, and should not even be entertained" (p. 210). Rather, he advocates injecting chance into the computer algorithms by using the theory of probability, the "only mathematical tool available to help map the unknown and the uncontrollable. The power of chance is widely underestimated," Mandelbrot asserts. "The physicists' concept of randomness is shaped by theories in which change is essential at the microscopic level, while at the macroscopic level it is insignificant. Quite to the contrary, in the case of the scaling random fractals that

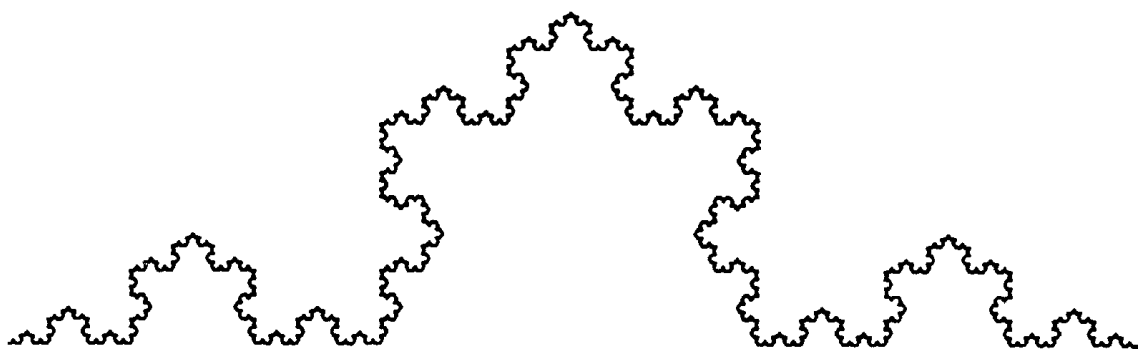

8. Making a Koch curve resemble a coastline. From Benoit B. Mandelbrot, The Fractal Geometry of Nature (New York, T983), pp. 43-44, by permission of W. H. Freeman Company. 
concern us, the importance of chance remains constant on all levels, including the macroscopic one" (p. 20r). Hence Mandelbrot's final figures are combinations of symmetries generated by deterministic algorithms and injections of chance caused by stochastic perturbations of the original algorithm.

The story of how Mandelbrot became interested in fractal geometry has the quality of a parable. He insists it originated with a book review "retrieved from a "pure' mathematician's wastebasket" (p. 422). His passion for resurrecting the forgotten and marginalized extends to people. In an appendix he gives brief biographies of earlier mathematicians who saw bits and pieces of what he would later make into a unified field. He sees them as avatars of himself, men who stepped out of their time to look at chaos in a new way. Their inclusion in his book fills a double role. On the one hand, they legitimate his enterprise by creating a tradition into which he can insert himself. On the other, their piecemeal accomplishments and peripheral status ensure that Mandelbrot can still claim for himself the honor of being the inventor of fractal geometry. The two editions of Fractal Geometry reveal the same kind of double gesture. In the first edition, when fractal geometry was a new idea, Mandelbrot took pains to present it as a continuation and extension of earlier ideas. By the second edition, when fractal geometry had become a recognized field that was attracting more researchers daily, Mandelbrot rewrote key passages to emphasize the uniqueness and singularity of his vision. There is an obvious political agenda to this jostling for position; it is no secret that Mandelbrot is a contender for a Nobel Prize.

Personal considerations notwithstanding, Mandelbrot's hunch that the most important contributions to chaos have been made by "mavericks" raises intriguing quesions about the relation of scientific content to style. Unlike many areas in the physical sciences, notably high-energy physics, chaos theory did not require expensive equipment operated by teams of researchers for its development. All it really required was an inclination to look at things from a different perspective. At least three of the field's superstars-Wilson, Feigenbaum, and Mandelbrot-see themselves as having made breakthroughs because they were interested in questions others had abandoned as unprofitable or suicidal. "The key seems to be time to 
spare," Mandelbrot writes; the sentiment is echoed by Feigenbaum and Wilson.

Probably most people in the physics community would agree that the style of chaologists is markedly different from the prevailing style in the community-more informal, eccentric, flamboyant. Is this style an outward and visible sign of a different way of thinking? And if so, is this new mode of thought merely the result of individual genius, as Mandelbrot implies, or it is in part culturally determined?

\section{Chaos and Culture: Deep Assumptions of the New Paradigms}

I remarked earlier on questions raised by models that do not take into account a system's specific mechanisms. Gleick reports that Bernardo Huberman met with incredulity when, at a conference on chaos, biology, and medicine, he demonstrated that a chaos model could account for erratic eye movements in schizophrenics (Gleick, 1987:227-230). A physiologist objected that Huberman had not taken into account the muscles responsible for moving the eye; a biologist pointed out that additional complexities were presented by the inertial effects of jelly within the eye. From these points of view, Huberman's model was a coincidence rather than an explanation. Huberman's critics thought that a proper explanation for erratic eye movements should show how the eye functioned as a unit and how it was connected to neural and muscular groups. The anecdote illustrates a point made by Thomas Kuhn (1970) in a different context: when a paradigm shift occurs, the very nature of what counts as an explanation changes. Explanations under two different paradigms are not just dissimilar; they are incommensurable.

In the chapters to come, I explore the nature of this paradigm shift and connect it with developments in critical theory, literature, technology, and culture. Its general outlines are already clear. Newtonian paradigms focus on individual particles or units. The assumption is that if these units are followed through time, their collective actions will add up to the system's behavior. The emphasis is therefore on isolating the appropriate unit and understanding the mecha- 
nisms that bind units into larger groups. It is a foundationalist approach, infused with assumptions about the integrity and autonomy of the individual.

The fundamental assumption of chaos theory, by contrast, is that the individual unit does not matter. What does matter are recursive symmetries between different levels of the system. Chaos theory looks for scaling factors and follows the behavior of the system as iterative formulae change incrementally. The regularities of the system emerge not from knowing about individual units but from understanding correspondences across scales of different lengths. It is a systemic approach, emphasizing overall symmetries and the complex interactions between microscale and macroscale levels. From this perspective, a proper explanation is one that is able to model largescale changes through the incremental evolution of a few iterative equations. Hence the emphasis in Huberman's model was not on the eye as such but on the equations that, when iterated, would produce wandering orbits equivalent to the eye's movements. From his point of view it was coincidental that the system in question was an eye. It could equally well have been fibrillation in the heart or fluctuations in cotton prices.

When one thinks about the great paradigm shifts of the past, one tends to suppose that the new paradigm was so obviously superior to the old that people were more or less instantly persuaded of its value. I suspect, however, that this was almost never the case. For people at the time, a new paradigm seemed to occlude as much as it revealed. It is only in retrospect that the occlusion seems insignificant and the revelation momentous, for once we are on the other side of the divide, we cease to care about the older issues. Today many scientists feel that chaos theory occludes essential aspects of a system's behavior; others believe that its revelations are immensely significant. It is difficult to adjudicate between these views, because they involve fundamental cultural assumptions that extend beyond the scope of scientific theory. Whether, for example, it is more important to decide if angels have enough corporeality to limit the number that can dance on the head of a pin, or to be able to describe mathematically how a cannonball's trajectory changes through time, depends on a multitude of cultural factors and not just on the merits of the theories alone. Scientific change is culturally conditioned. 
To illustrate how arguments for the autonomy of science are culturally based, I should like to turn to a text I have referred to throughout this study, Gleick's Chaos. Although Chaos is written to be accessible to a general audience, it is no facile popularization. Meticulously researched, it draws from hundreds of personal interviews and cites many of the major technical articles in the field. It is also beautifully written, combining a journalist's eye for human interest with a scientist's interest in concepts. I recommend it to anyone interested in the subject. I certainly learned a great deal from it, and I am grateful to the author for making it available to me before its publication.

Gleick describes his book as a narrative history. He rightly sees it as telling a story, and like any storyteller, he has shaped his material in ways both obvious and subtle. It is this shaping that I want to explore, for it depicts the discovery of chaos as essentially an individual enterprise, unaffected by the culture around it. I shall begin my story about Gleick's story at the point where it began for mewhen, on a second reading, I realized that this book has no women in it. Hundreds of men are mentioned by name; some dozen are depicted in enough detail so that one almost feels one knows them. But no women, or virtually none, appear. ${ }^{14}$ (Another reading revealed that women do appear a few times in the text, but only as anonymous "wives" who accompany their scientist-husbands at social events. In one case, Gleick implies that their presence transformed the meeting of two scientists into a social occasion, making it impossible for the men to conduct serious scientific conversation [p. I 4 I].) I had a divided response to this absence of women. On the one hand, Gleick can scarcely invent them where they do not exist. Perhaps even more than most sciences, chaos is heavily dominated by men, especially in America. On the other hand, the exclusion of women in Gleick's text goes beyond the acknowledged scarcity of

\footnotetext{
${ }^{14}$ Several women are listed in the references. They include those whose names appear as co-authors of scientific papers; Erica Jen as a respondent (260); and Mary Lucy Cartwright as a researcher in chaotic oscillators (272). Evelyn Fox Keller has a fine analysis of masculinist assumptions in science in Reflections on Gender and Science (New Haven: Yale University Press, r985). See also Ruth Bleier, Science and Gender: A Critique of Biology and Its Theories on Women (Oxford and New York: Pergamon, 1984), and Sandra Harding, The Science Question in Feminism (Ithaca: Cornell University Press, I986).
} 
distinguished women scientists. It pervades the entire depicted world.

The absence of women is most striking in the personal vignettes that Gleick uses to punctuate his explanations of scientific concepts. These vignettes show what the living quarters of this or that scientist are like; they reveal where one scientist goes for walks and what another likes to eat. But in all this richly textured detail, women never appear. The impression is that none of these men has a relationship with a woman which is important in his intellectual life; none works with a female collaborator who is an important contributor; and none spends much time with women. Having noticed this, I also began to notice the settings in which the scientists appeared. Very often they emphasize the solitary nature of their intellectual lives. Feigenbaum is shown walking away from a group so he can observe a waterfall; on another occasion, a crucial insight comes when he walks away from a group outdoors and notices that their talk is reduced to babble by the distance. Another scientist likes to take long walks in the desert, sometimes leaving his family for weeks at a time. When living quarters are discussed, they are depicted as eccentric or antidomestic. Feigenbaum has no furniture; the Santa Cruz collective lives in a house littered with bean bag chairs. Food is equally rudimentary or odd.

No doubt these details are accurate. But by mentioning them and not others, Gleick creates an ambiance for the scientific discoveries he describes. As it is shown here, the world of science is first of all genderless-genderless because there is only one gender. It is also solitary, with chance connections made between individuals who discover, often quite by accident, that someone else somewhere in the world is working on the same problem they are. And it is marked by a flow of narrative time in which certain moments are retrospectively identified as decisive, even though they may have seemed ordinary enough when they occurred. Treating time in this way is effective in creating the kind of suspense that keeps readers turning pages; but it also makes time into a series of agons marking the junctures at which fate took a different turn. These components work together to substantiate Gleick's claim that "no mass of scientists" brought about a new paradigm, only "a few solitary individuals" (p. I 52 ).

Once I noticed how the material was shaped, I began to wonder 
how it fitted into the larger view of chaos that Gleick presents. For many of the scientists whose words he records, chaos is more than just another theory. It represents an opening of the self to the messiness of life, to all the chaotic unpredictable phenomena that linear science taught these scientists to screen out. Once roused, they remember that the messiness was always there. Moreover, now they are able to see nonlinearity in a new light, perceiving it as central rather than marginal, beautiful rather than aberrant. Chaotic unpredictability and nonlinear thinking, however, are just the aspects of life that have tended to be culturally encoded as feminine. Indeed, chaos itself has often been depicted as female. In the English Renaissance, for example, the male seed was commonly represented as contributing form; the female was thought to contribute raw unshaped materia, matter devoid of form or structure. In validating chaos as a scientific concept, Gleick seems to have found it necessary to expunge the female from his world. Why?

I can of course only speculate about the psychological and cultural dynamics underlying this exclusion. Nevertheless, certain aspects are sufficiently clear as to be almost obvious. In the Western tradition, chaos has played the role of the other-the unrepresented, the unarticulated, the unformed, the unthought. In identifying with chaos, the scientists that Gleick writes about open themselves to this otherness, and they perceive their intercourse with it as immensely fructifying-for their work, for their disciplines, and for them personally. But otherness is also always a threat, arousing the desire to control it, or even more extremely to subsume it within the known boundaries of the self, thus annihilating the very foreignness that makes it dangerously attractive. Both of these impulses are evident in Gleick's text, and probably are at work within chaos theory as well. The desire to control chaos is evident in the search for ways to rationalize it. By finding within it structures of order, these scientists have in effect subsumed chaos in the familiar. But if this incorporation were entirely successful, chaos could no longer function in its liberating role as a representation of the other. Perhaps this is why Mandelbrot goes out of his way to argue against the complete rationalization of chaos; he believes that some residue of the untamable and nonrational should always remain.

This ambivalence toward chaos is encoded into Gleick's text, especially in the divided response toward the feminine. Representa- 
tions of actual women and of activities closely associated with them are rigorously excluded from the depicted world. Paradoxically, this exclusion facilitates the incorporation of the feminine principle of chaos into science. By admitting the feminine as an abstract principle but excluding actual women, Gleick attains control over the polysemy of chaos, striping it of its more dangerous and engendered aspects. As a result, chaos is admitted into the boundaries of scientific discourse, but science remains as monolithically masculine as ever.

In achieving this accommodation, Gleick's text engenders a series of paradoxes. It depicts chaos theory as the achievement of extraordinary individuals who stepped out of the mainstream; but this scenario of the solitary man who opens up a frontier is itself deeply a part of the American mainstream. It shows science as an exclusively male domain; but it is the peculiar project of this domain to have intercourse with a feminine principle. It intimates that scientific discovery is an activity that men engage in when they separate themselves from their families and from the larger culture; but the theories these men formulate imply that the individual unit is not important. The complex play of gender, individuality, and scientific theory in Gleick's text suggests that chaos theory is a deeply fissured site within the culture, in which lingering assumptions from older paradigms are embedded within the emerging paradigms of the new science. When there is this kind of complex interplay between science and culture, science cannot be separated from the cultural matrix. Like literature, science is always already cultural and cannot be otherwise.

I do not believe that the scientists Gleick writes about acted in isolation. I think that they rather acted like lightning rods in a thunderstorm or seed crystals in a supersaturated solution. They gave a local habitation and a name to what was in the air. It was because the cultural atmosphere surrounding them was supercharged that these ideas seemed so pressing and important. Mandelbrot, though he clearly wants to claim the territory for himself, recognizes that earlier mathematicians had penetrated his domain. Because the time was not right, their forays were regarded as dead ends. Now, for reasons that are as complex as the chaotic systems the new paradigms represent, the time is right. 